\title{
Culture, group membership, and face recognition. Commentary: Will you remember me? Cultural differences in own-group face recognition biases
}

\author{
Kai Kaspar* \\ Department of Psychology, University of Cologne, Cologne, Germany
}

Keywords: cross-cultural comparison, group membership, face recognition, ingroup bias, prioritization

\section{A commentary on}

Will you remember me? Cultural differences in own-group face recognition biases by Ng, A. H., Steele, J. R., and Sasaki, J. Y. (2016). J. Exp. Soc. Psychol. 64, 21-26. doi: 10.1016/j.jesp.2016.01.003

Literature reports that same-race faces are better recognized than cross-race faces. This cross-race effect has been observed, inter alia, in European Americans (MacLin et al., 2004) as well as in Asian Americans and East Asians (Michel et al., 2006; Hayward et al., 2008). Although research showed that cross-race effects reflect a superiority in the processing of components and configurations of own-race faces (Hayward et al., 2008), empirical evidence showed that (arbitrary) social categorization similarly contributes to an ingroup bias in face recognition (MacLin and Malpass, 2001). This finding suggests that mere social categorization is sufficient to bias face recognition performance in favor of ingroup faces, reflecting a motivational preference to individuate ingroup members (Hugenberg et al., 2010).

Following these findings, Ng et al. (2016) examined whether participants' cultural background moderates the effect of arbitrarily determined social group membership on the recognition performance for ingroup/outgroup targets displaying neutral facial expressions. According to their focal hypothesis, North Americans should preferentially define their ingroups on broader social categories, whereas East Asians should define their ingroups with a focus on preexisting relationships established through friendship and family (Brewer and Yuki, 2007). Therefore, $\mathrm{Ng}$ and colleagues expected that European Canadians, but not East Asians, would show an ingroup bias in face recognition when group membership is arbitrarily determined by a minimal group manipulation: A color-coding system indicated whether one's own personality fits the personality of a displayed person (Study 1) or whether one's preexisting university affiliation fits that of a displayed person (Study 2). Same colors indicated ingroup faces, different colors indicated outgroup faces. In both studies, European Canadians recognized previously observed ingroup faces better than outgroup faces. East Asian Canadians did not show this bias for arbitrarily determined ingroup faces. Hence, the authors concluded that culture moderates the effect of mere social categorization on face recognition. Overall, these studies provide a valuable extension of previous research by introducing a cross-cultural perspective. However, some crucial aspects should be considered in future research in this field.

Kaspar K (2016) Culture, group membership, and face recognition. Commentary: Will you remember me?

\section{THE EMPIRICAL ASPECT}

The result pattern for Asian Canadians showed some inconsistency across studies. European Canadians recognized White faces better than East Asian faces in both studies. In contrast, Asian 
Canadians showed a better memory for Asian faces in Study 1 (personality), whereas they better recognized White faces in Study 2 (university affiliation), suggesting a culture-specific effect of group membership. Generally, when observing and memorizing a sequence of many faces, one's cognitive capacities are quickly exceeded, so one has to prioritize the targets. In the absence of other veridical information about a target's relevance we usually refer to contextual information to reduce situational uncertainty (Kaspar, 2013; Kaspar and Krull, 2013) and ambiguity (Kaspar, in press). Correspondingly, color as a contextual sign for one's university affiliation might have helped to identify and separate targets of high (ingroup) and low (outgroup) priority in European Canadians, reflected in a better recognition of ingroup faces. In contrast, social categorization in terms of university affiliation might have primed thoughts of acculturation processes in Asian Canadians instead. Given the central argument of motivationally driven prioritization of visual input (Hugenberg et al., 2010), it is conceivable that the first-generation Asian Canadians tested by $\mathrm{Ng}$ and colleagues have been sensitized toward acculturation processes, leading to an increased motivation to accurately process White faces representing the host culture's population. In contrast, when color indicated the personality type (Study 1), such cognitive processes were rather unlikely and hence cross-race effects were visible in both European and Asian Canadians. Consequently, in order to verify the validity of the reported results, the study should be replicated with Asians living in a respective country without a personal involvement in acculturation processes. More generally, it should be considered that test-taking behavior can be influenced by participants' relation to the culture in which the test is administered (Cofresi and Gorman, 2004).

\section{THE METHODOLOGICAL ASPECTS}

The study by $\mathrm{Ng}$ and colleagues shares one methodological limitation with several other studies investigating group effects on face recognition performance, because it remains open whether group membership affects cognitive processes in the

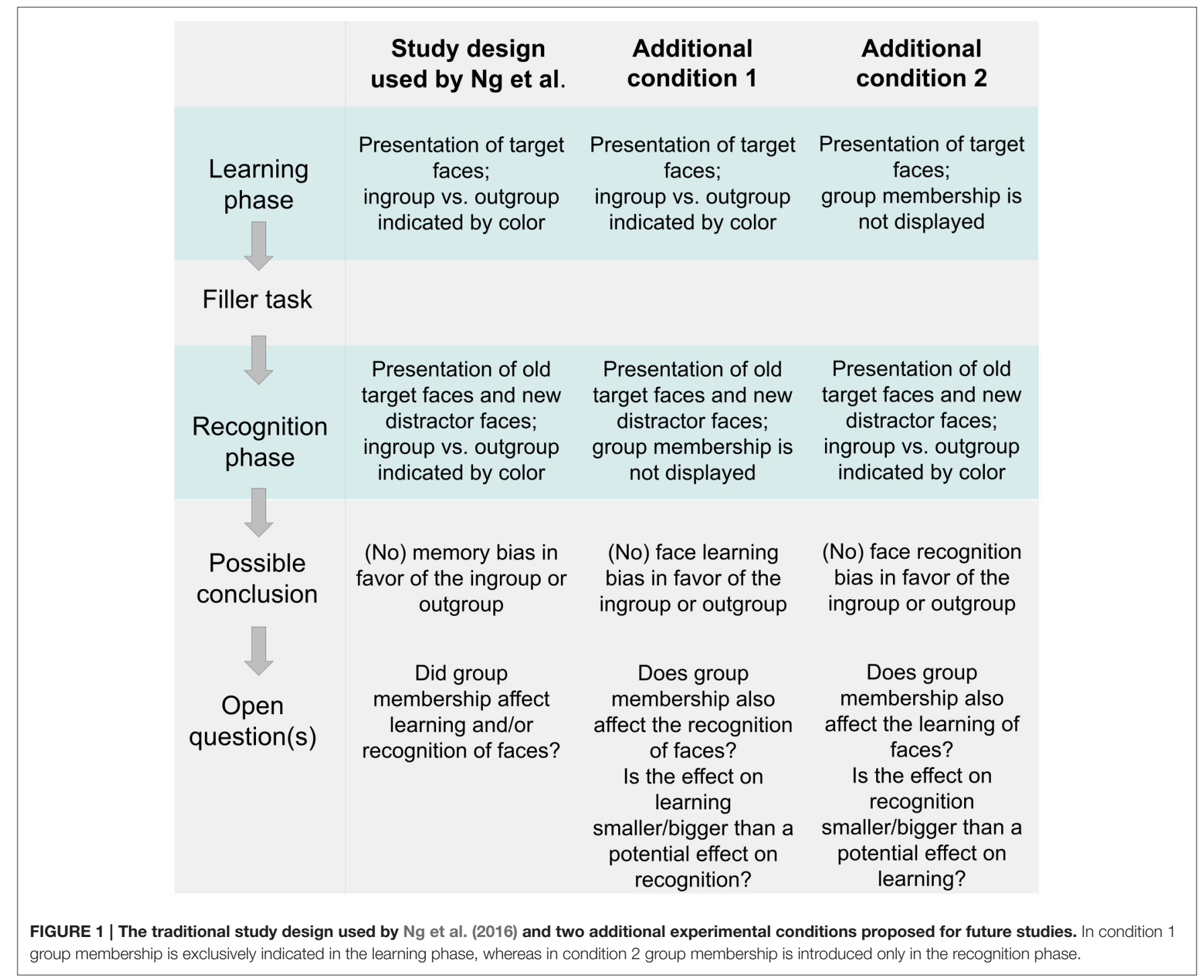


learning or/and recognition phase. In addition to the judgmental responses investigated by $\mathrm{Ng}$ et al., other research suggests that the recognition bias for ingroup faces reflects a motivationally driven top-down modulation of cognitive processes, leading to an increased activity of the fusiform face area in the recognition phase (Van Bavel et al., 2011). Thus, group membership might affect selective attention and cognitive processing in terms of encoding face attributes when observing target faces in the learning phase. Alternatively, group membership might (also) increase the motivation to thoroughly compare memory content with current visual input in the recognition phase. To compare these alternatives, future studies should extend the traditional study design used by $\mathrm{Ng}$ et al. by two additional conditions, as illustrated in Figure 1.

Furthermore, it is important to pay attention when selecting face stimuli for cross-cultural comparisons. Previous studies revealed that attractive faces can intensify approach motivation (Zebrowitz et al., 2015) and that face recognition performance may depend on the emotion displayed (Di Domenico et al., 2015; Righi et al., 2015). Given cultural differences in the judgment of facial attractiveness (Sorokowski et al., 2013) and in the interpretation of emotional facial expressions (Yuki et al., 2007), the attractiveness and emotional valence

\section{REFERENCES}

Brewer, M. B., and Yuki, M. (2007). "Culture and social identity," in Handbook of Cultural Psychology, eds S. Kitayama and D. Cohen (New York, NY: Guilford Press), 307-322.

Cofresi, N. I., and Gorman, A. A. (2004). Testing and assessment issues with Spanish-English bilingual Latinos. J. Couns. Dev. 82, 99-106. doi: 10.1002/j.1556-6678.2004.tb00290.x

Di Domenico, A., Palumbo, R., Mammarella, N., and Fairfield, B. (2015). Aging and emotional expressions: is there a positivity bias during dynamic emotion recognition? Front. Psychol. 6:1130. doi: 10.3389/fpsyg.2015.01130

Hayward, W. G., Rhodes, G., and Schwaninger, A. (2008). An own-race advantage for components as well as configurations in face recognition. Cognition 106, 1017-1027. doi: 10.1016/j.cognition.2007.04.002

Hugenberg, K., Young, S. G., Bernstein, M. J., and Sacco, D. F. (2010). The categorization-individuation model: an integrative account of the other-race recognition deficit. Psychol. Rev. 117, 1168-1187. doi: 10.1037/a0020463

Kaspar, K. (2013). Embodied cognition is a weighty matter: heaviness influences the perception of disease severity, drug effectiveness, and side effects. PLOS ONE 8:e78307. doi: 10.1371/journal.pone.0078307

Kaspar, K. (in press). Arousal-biased preferences for sensory input: an agent-based and multisource perspective. Behav. Brain Sci.

Kaspar, K., and Krull, J. (2013). Incidental haptic stimulation in the context of flirt behavior. J. Nonverbal Behav. 37, 165-173. doi: 10.1007/s10919-013-0154-0

MacLin, O. H., and Malpass, R. S. (2001). Racial categorization of faces: the ambiguous race face effect. Psychol. Public Pol. L. 7, 98-118. doi: 10.1037/10768971.7.1.98

MacLin, O. H., Van Sickler, B. R., MacLin, M. K., and Li, A. (2004). A reexamination of the cross-race effect: the role of race, inversion, and basketball trivia. N. Am. J. Psychol. 6, 189-204.

Michel, C., Rossion, B., Han, J., Chung, C. S., and Caldara, R. (2006). Holistic processing is finely tuned for faces of one's own race. Psychol. Sci. 17, 608-615. doi: 10.1111/j.1467-9280.2006.01752.x of same-race and other-race faces have to be validated by all investigated culture groups to prevent potential confounds.

\section{THE CONCEPTUAL ASPECT}

Finally, let us assume the validity of $\mathrm{Ng}$ et al.'s focal hypothesis stating that Asians preferentially define their ingroups by preexisting relationships established through friendship and family. If so, it might be that we will nonetheless find ingroup favoritism regarding face recognition in Asians when a minimal group manipulation is applied to a set of faces sampled from the pool of the participant's family or group of friends. Indeed, such a "second order" effect appears to be an inherent part of the assumption that ingroup membership is initially determined by personal relationships in (some) Asian cultures, while this does not make the prioritization of multiple targets superfluous in the face of strongly limited cognitive capacities.

\section{AUTHOR CONTRIBUTIONS}

The author confirms being the sole contributor of this work and approved it for publication.

Ng, A. H., Steele, J. R., and Sasaki, J. Y. (2016). Will you remember me? Cultural differences in own-group face recognition biases. J. Exp. Soc. Psychol. 64, 21-26. doi: 10.1016/j.jesp.2016.01.003

Righi, S., Gronchi, G., Marzi, T., Rebai, M., and Viggiano, M. P. (2015). You are that smiling guy I met at the party! Socially positive signals foster memory for identities and contexts. Acta Psychol. 159, 1-7. doi: 10.1016/j.actpsy.2015.05.001

Sorokowski, P., Kościński, K., and Sorokowska, A. (2013). Is beauty in the eye of the beholder but ugliness culturally universal? Facial preferences of Polish and Yali (Papua) people. Evol. Psychol. 11, 907-925. doi: 10.1177/147470491301100414

Van Bavel, J. J., Packer, D. J., and Cunningham, W. A. (2011). Modulation of the fusiform face area following minimal exposure to motivationally relevant faces: evidence of in-group enhancement (not out-group disregard). J. Cogn. Neurosci. 23, 3343-3354. doi: 10.1162/jocn_a_00016

Yuki, M., Maddux, W. W., and Masuda, T. (2007). Are the windows to the soul the same in the East and West? Cultural differences in using the eyes and mouth as cues to recognize emotions in Japan and the United States. J. Exp. Soc. Psychol. 43, 303-311. doi: 10.1016/j.jesp.2006.02.004

Zebrowitz, L. A., Franklin, R. G., and Palumbo, R. (2015). Ailing voters advance attractive congressional candidates. Evol. Psychol. 13, 16-28. doi: $10.1177 / 147470491501300102$

Conflict of Interest Statement: The author declares that the research was conducted in the absence of any commercial or financial relationships that could be construed as a potential conflict of interest.

Copyright (C) 2016 Kaspar. This is an open-access article distributed under the terms of the Creative Commons Attribution License (CC BY). The use, distribution or reproduction in other forums is permitted, provided the original author (s) or licensor are credited and that the original publication in this journal is cited, in accordance with accepted academic practice. No use, distribution or reproduction is permitted which does not comply with these terms. 